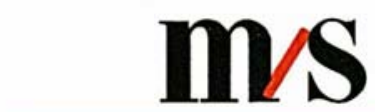

médecine/sciences $1992 ; 8: 205-7$

\section{HTLV-1 1992, ASPECTS MOLÉCULAIRES ET IMMUNO-PRÉVENTION}

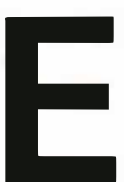

n 1987, nous évoquions, dans médecine/sciences, les aspects cliniques et épidémiologiques de l'HTLV-1 et des affections associées, leucémie T de l'adulte (ATL) et neuromyélopathie chronique (tropical spastic paraparesis/HTLV1 associated myelopathy, TSP/HAM) [1].

Où en sommes-nous en 1992 au niveau de la connaissance du virus, des relations avec l'hôte et de sa prévention?

La structure du génome de l'HTLV-1, qui semblait relativement simple comparée à celle des VIH, tend à se compliquer. Outre ses deux protéines régulatrices, Tax et Rex, d'autres protéines codées par l'HTLV-1 viennent d'être mises en évidence, par la découverte, grâce au séquençage des produits d'amplification d'ADN complémentaire d'HTLV-1, de nouveaux ARN messagers ([2, 3], Koralnik et al., en préparation). Ceux-ci, engendrés par des sites d'épissage jusqu'alors inconnus, présents dans la région $\mathrm{pX}$, codent pour des protéines nucléaires dont la fonction dans le cycle viral et la genèse des processus pathologiques associés à l'HTLV-1 sont actuellement à l'étude. Ainsi l'HTLV-1, comme le VIH, a-t-il développé des mécanismes posttranscriptionnels lui permettant d'accroître la complexité fonctionnelle de son génome.

Le récepteur cellulaire de l'HTLV-1, encore inconnu, devrait être une molécule relativement ubiquitaire puisque ce virus peut infecter in vitro un grand nombre de types cellulaires chez l'animal (lapin, rat...) et chez l'homme. L'équipe de Robin Weiss à Londres a montré que le gène codant pour le récepteur de l'HTLV-1, mais aussi du HTLV-2, était situé sur le chromosome 17. L'expression virale dans les cellules mononucléées circulantes des sujets infectés asymptomatiques ou ayant une TSP/HAM et dans les cellules tumorales d'ATL, était considérée jusqu'à présent comme nulle. En effet, aucun ARN messager viral, ni expression protéique, ni particules rétrovirales n'étaient détectables dans ces cellules in vivo, par les techniques classiques. Récemment, plusieurs équipes [4-6] grâce à l'utilisation de techniques plus sensibles telles que l'hybridation in situ et l'amplification génomique in vitro (PCR), ont pu détecter une telle expression virale in vivo dans une proportion très faible $(0,02 \%$ à $1 \%)$ des cellules mononucléées des sujets infectés porteurs d'une charge provirale importante. Dans les TSP/HAM où 5 à $30 \%$ des lymphocytes CD4 circulants ont un provirus HTLV-1 intégré de façon polyclonale, une cellule sur 5000 environ est le siège d'une activité transcriptionnelle virale [6]. Il reste par ailleurs accepté que les cellules tumorales d'ATL ne sont pas le siège d'une expression virale in vivo.

Le rôle de la protéine Tax dans le processus de prolifération cellulaire in vivo a été démontré. De même, la prolifération lymphoïde spontanée in vivo caractéristique des TSP/HAM et des sujets HLTV-1 séropositifs porteurs chroniques serait en partie relayée par Tax [4].

Après mise en culture de ces cellules in vitro, la rapidité de la réplication virale, en quelques heures, contraste avec la situation in vivo et suggère l'existence de facteurs sériques et/ou de lymphokines contrôlant la latence virale HTLV-1 in vivo.

Au niveau immuno-virologique, la plupart des équipes s'accordent sur le parallélisme, presque absolu, entre détection du génome proviral par PCR et présence d'anticorps sériques. Cependant, la présence éventuelle d'une infection virale dans des sites immunoprotégés tels que le système nerveux central, qui n'entraînerait pas de réponse immunitaire et échapperait ainsi 
à la détection sérologique et par PCR dans les cellules mononucléées du sang circulant, demeure. Une telle possibilité devrait être explorée chez les malades TSP/HAM, les enfants de mères séropositives et dans les fratries de ces patients.

La leucémogenèse ATL serait un processus à étapes multiples dans lequel l'HTLV-1 apparaît avant tout comme un agent initiateur causant une prolifération polyclonale de cellules $\mathrm{CD}^{+}{ }^{+}$à partir de laquelle un deuxième événement génétique, indépendant du virus, entraînerait une sélection monoclonale de cellules préleucémiques ou leucémiques. Le rôle de l'HTLV-1 dans la persistance du processus leucémique est plus discutable. Concernant la genèse des TSP/HAM, les cellules T cytotoxiques restreintes aux molécules HLA de classe II et dirigées contre certains épitopes des protéines régulatrices virales pourraient jouer un rôle clé [7]. Les causes de la rareté (2 à $10 \%)$ de l'émergence de ces affections parmi les sujets infectés restent inconnues mais suggèrent l'intervention déterminante de cofacteurs génétiques et/ou liés à l'environnement. $\mathrm{Au}$ niveau épidémiologique, les régions les plus touchées sont toujours les Caraïbes et les régions avoisinantes d'Amérique du Nord et du Sud, l'Afrique intertropicale, et les îles du sud de l'archipel nippon. Une région du Moyen-Orient (Iran) et la Mélanésie, ont rejoint les zones de forte endémie virale. On évalue actuellement entre 15 et 20 millions le nombre de sujets infectés par l'HTLV-1 dans ces régions où 1 à $30 \%$ de la population générale possède des anticorps sériques. La transmission virale de la mère à l'enfant par l'allaitement domine la scène en région endémique, la transmission sexuelle prenant le relais et augmentant le réservoir au cours de la vie, en particulier chez la femme. En Occident, les groupes à risques sont les toxicomanes par voie intraveineuse, lesquels sont infectés aussi bien par l'HTLV-1 (2 à $7 \%$ aux USA) que surtout par l'HTLV-2 $(0,5$ à $30 \%$ aux USA), ce dernier virus ayant $65 \%$ d'analogie nucléotidique avec l'HTLV-1. On peut se demander si cette observation dans un groupe limité ne risque pas d'être à l'origine d'une épidémie en Occident, comme cela a été le cas au début pour le VIH. A partir de ce réservoir, une transmission sexuelle, certes moins efficace pour les HTLV-1 et -2 que pour le VIH, pourrait se développer dans la population générale. A ce jour, on ne connaît qu'une seule population naturellement endémique pour l'HTLV-2 : certains groupes d'Amérindiens du Panama, de Colombie, d'Amazonie brésilienne et des États-Unis. Ainsi, si l'HTLV-1 apparaît comme un rétrovirus humain de l'Ancien Monde, l'HTLV-2 serait celui du Nouveau Monde.

Au niveau clinique, d'autres syndromes ont été associés à l'HTLV-1, sans que le lien causal n'ait encore été établi. Il s'agit surtout de polymyosites, d'arthrites mais aussi d'exocrinopathies de type syndrome de Goujerot-Sjögren*, de pneumopathies interstitielles lymphoïdes infracliniques et de dermatites infectieuses surtout chez les jeunes enfants HTLV-1 séropositifs dans les Caraïbes.

Faisant le lien entre aspect fondamental et épidémiologie de terrain, l'épidémiologie moléculaire de l'HTLV-1 a été récemment développée par plusieurs équipes, dont la nôtre [8-10]. Ces études ont montré que la variabilité génomique de l'HTLV-1 est très faible, de l'ordre de $0,5 \%$ à $3 \%$ selon les isolats et les régions du génome étudiées, cela étant surtout le fait de mutations ponctuelles (substitutions). D'autre part, il existe une grande stabilité d'un virus donné au cours du temps chez un même individu et chez différents hôtes successifs, ce qui contraste avec la plasticité génomique du VIH. Par ailleurs, les virus provenant de mêmes régions géographiques montrent des identités moléculaires, quel que soit le statut clinique de l'individu d'où provient le virus (sujet sain, ATL ou TSP/HAM). Ainsi les virus de la région du centre du Zaïre sont-ils génétiquement plus proches l'un de

- Syndrome de Gougerot-Sjögren : considéré comme étant le plus souvent d'origine auto-immune, ce syndrome se caractérise par un tarissement des sécrétions exocrines, principalement lacrymales et salivaires. Il accompagne généralement un rhumatisme inflammatoire. l'autre que ceux de l'Afrique de l'Ouest ou du Japon. L'étude de certains isolats de populations a permis de découvrir la présence de variants moléculaires de l'HTLV-1 distants de l'HTLV-1 prototype (5 à $15 \%$ de divergence selon les gènes), en Mélanésie [11] et chez les aborigènes australiens. L'analyse du polymorphisme des fragments de restriction du LTR de 30 provirus HTLV-1 permet de définir trois sous-types géographiques [12]. Il semble donc qu'il existe des variants moléculaires de l'HTLV-1 attachés à tel ou tel groupe humain et à ses migrations spacio-temporelles [10]. En revanche, à ce jour, aucune équipe n'a pu démontrer l'existence de séquences spécifiques d'une symptomatologie donnée (ATL ou TSP/HAM) [8, 10, $13,14]$. L'utilisation dans des modèles animaux de clones infectieux portant ces diverses mutations, ainsi que de souris transgéniques et de techniques analytiques pour l'étude de la transactivation des LTR, est nécessaire pour donner un sens fonctionnel, une signification biologique à certaines mutations ponctuelles observées dans différents isolats de populations avec des cas de TSP/HAM, d'ATL ou des sujets sains HTLV-1 séropositifs. Une telle étude pourrait montrer une spécialité, soit hématologique, soit neurologique. Ce sujet est développé dans l'article de Pascale Briand ( $p .208$ de ce numéro).

La thérapeutique des affections liées à l'HTLV-1 est encore décevante malgré divers essais associant chimiothérapie-antivirauximmunothétapie dans les ATL et immunomodulateurs-corticothérapieantiviraux dans les TSP/HAM. L'amélioration clinique est avant tout transitoire et à court terme. Comment limiter la transmission de ce virus ? Dès 1986, le Japon établissait le dépistage HTLV-1 systématique des dons du sang, ce qui a entraîné deux ans plus tard une diminution de l'incidence des maladies neurologiques liées à ce virus de l'ordre de $15 \%$. Aux États-Unis, ce dépistage systématique dans les centres de la Croix Rouge a été mis en place dès 1988. Il ne l'est que depuis juillet 1991 en France métropolitaine, précédé par plusieurs cas de TSP/HAM 
post-transfusionnels diagnostiqués en France dès 1990 [15].

Afin d'éviter la transmission chez les toxicomanes par voie intraveineuse, une éducation sanitaire et la fourniture d'aiguilles deviennent indispensables.

La transmission par l'allaitement a conduit au Japon, dès 1985, à informer des mères HTLV-1 séropositives, leur demandant de ne pas allaiter leur enfant. Ce qui est tout à fait possible et sans danger pour l'enfant au Japon est impossible et serait très dangereux dans les pays africains et en Amérique du Sud.

Par ailleurs, l'excellente stabilité génomique de ce virus est favorable au développement d'un vaccin, de même que l'existence de modèles expérimentaux chez le lapin. On a pu ainsi démontrer que des vaccins recombinants et, même des gammaglobulines humaines (provenant de donneurs HTLV-1 séropositifs), protégeaient les lapins d'une infection ultérieure par l'HTLV-I, soit par voie sanguine, soit par allaitement. Le développement d'un vaccin contre l'HTLV-1 se heurte au fait que ce sont surtout les pays en voie de développement qui sont touchés et que seulement 2 à $10 \%$ des personnes infectées développent une affection grave alors que dans le cas du VIH, c'est encore l'immense majorité des gens infectés qui développent un SIDA.

Quel type de vaccin est souhaitable? Un vaccin recombinant vivant pourrait utiliser comme vecteur, la vaccine, le BCG, un adénovirus, ou encore une salmonelle. Ces deux derniers vecteurs permettraient un vaccin oral qui serait d'une distribution plus facile qu'un vaccin injectable dans les pays en voie de développement. L'induction d'une immunité au niveau des muqueuses aérodigestives supérieures et génitales pourrait servir de modèle pour le VIH où la nécessité en est particulièrement évidente.

En conclusion, le développement des recherches fondamentales en biologie moléculaire, immuno-virologie, épidémiologie et clinique humaine permettent d'entrevoir la possibilité d'une éradication par vaccin de cet oncorétrovirus humain, et par là de certaines leucémies et syndromes neuro-dégénératifs

\section{RÉFÉRENCES}

1. Gessain A, de Thé G. Virus HTLV-1, leucémies $\mathrm{T}$ de l'adulte et neuromyélopathies chroniques. médecine/sciences $1987 ; 3: 471-8$.

2. Berneman Z, Gartenhaus R, Reitz M, et al. Expression of alternatively spliced human $T$ lymphotropic virus type 1 (HTLV-1) pX mRNA in infected cell lines and primary uncultured cells from patients with adult T-cell leukemia/lymphoma and healthy carriers. Proc Natl Acad Sci USA 1992 (sous presse)

3. Ciminale V, Pavlakis G, Derse D, Cunningham C, Felber B. Complex splicing in the HTLV family of retroviruses : novel mRNAs and proteins produced by HTLV-1. J Virol 1992 (sous presse).

4. Tendler CL, Greenberg SJ, Blattner WA, et al. Transactivation of interleukine 2 and its receptor induces immune activation in human $\mathrm{T}$-cell lymphotropic virus type 1 associated myelopathy : pathogenic implications and rationale for immunotherapy. Proc Natl Acad Sci USA 1990; 87 : 5218-22.

5. Beilke MA, Riding ID, Gravel M, et al. In situ hybridization detection of HTLV-1 RNA in peripheral blood mononuclear cells of TSP/HAM patients and their spouses. J Med Virol 1991; 33 : 64-71.

6. Gessain A, Louie A, Gout O, Gallo RC, Franchini G. Human T cell leukemia/lymphoma virus type 1 expression in fresh peripheral blood mononuclear cells from patients with tropical spastic paraparesis/HTLV-1 associated myelopathy. $J$ Virol $1991 ; 65: 1628-33$.

7. Jacobson S, Shida H, McFarlin De, Fauci AS, Koenig S. Circulating CD8 ${ }^{+}$cytoxic T lymphocytes specific for HTLV-1 pX in patients with HTLV-1 associated neurological disease. Nature 1990 ; 348 : 245-8.

8. Komurian F, Pelloquin F, de Thé G. In vivo genomic variability of human T-cell leukemia virus type 1 depends more upon geography than upon pathologies. J Virol 1991 ; 65 : 3770-8.

9. Schulz TF, Calabro ML, Hoad JG, et al. HTLV-1 enveloppe sequences from Brazil, the Caribbean, and Romania : clustering of sequences according to geographic origin and variability in an antibody epitope. Virology $1991 ; 184: 483-91$. 10. Gessain A, Gallo RC, Franchini G. The low degree of HTLV-1 genetic drift in vivo as a mean to follow viral transmission and movement of ancient human population. J Virol 1992 (sous presse).

11. Gessain A, Yanagihara R, Franchini G, et al. Highly divergent molecular variants of human $\mathrm{T}$ lymphotropic virus type 1 from isolated populations in Papua New Guinea and the Solomon Islands. Proc Natl Acad Sci USA 1991 ; 88 : 7694-8.

12. Komurian-Pradel F, Pelloquin F, Sonoda S, de Thé G. Geographical Subtypes demonstrated by RFLP following PCR in the LTR region of HTLV-1. AIDS Res Hum Retrovir 1992 (sous presse). 13. Daenke S., Nightingale S, Cruickshank JK, Bangham RM. Sequence variants of human T-cell lymphotropic virus type 1 from patients with tropical spastic paraparesis and adult T-cell leukemia do not distinguish neurological from leukemic isolates. J Virol 1990 ; 64 : 1278-82.

14. Kinoshita T, Tsujimoto A, Shimotohno K. Sequence variations in LTR and env regions of HTLV-1 do not discriminate between the virus from patients with HTLV-1 associated myelopathy and adult T-cell leukemia. Int J Cancer 1991; 47 : 491-5.

15. Gout O, Baulac M, Gessain A, et al. Rapid development of myelopathy after HTLV-1 infection acquired by transfusion during cardiac transplantation. N Engl J Med 1990 ; 322 : 383-8. 